\title{
CÁLCULO DE SERIES ARMÓNICAS DE RIEMANN CON EXPONENTE PAR
}

\author{
CALCULATE OF HARMONIC SERIES RIEMANN WITH EVEN EXPONENT
}

Jorge, Morales Paredes

Matemático, Profesor cátedra, Departamento de matemáticas, Universidad Militar Nueva Granada, Bogota, Colombia.

Weimar, Muñoz Villate

Matemático, Profesor cátedra, Departamento de matemáticas, Universidad Militar Nueva Granada, Bogota, Colombia.

Solón E., Losada Herrera

M.Sc., Profesor tiempo completo, Departamento de matemáticas, Universidad Militar Nueva Granada, Bogotá, Colombia.

Fecha de recepción: 8 agosto de 2007

Fecha de aprobación: 16 de junio de 2008

\section{RESUMEN}

La llamada función Zeta de Riemann fue introducida por Euler mediante la definición $\xi(z)=\sum_{n=1}^{\infty} \frac{1}{n^{2}}$, que se trata de una serie convergente en la que $z$ es un número complejo con parte real mayor que uno. El presente trabajo va encaminado a presentar una fórmula recurrente para el cálculo de series $\sum_{n=1}^{\infty} \frac{1}{n^{2 k}}$, para $k \in Z^{+}$. Es conocido que Euler desarrolló este mismo caso particular, trabajando con los ceros de la función zeta ${ }_{[3]}$, nosotros realizamos dicho cálculo utilizando la función cot $z$ e inducción matemática. Para la comprensión de este escrito, es necesario que el lector tenga algunas nociones de variable compleja, como son: función analítica, expansión en serie de Taylor, series de Laurent, entre otros [1], [2].

Palabras claves: Serie de Laurent, división larga, números de Bernoulli e inducción matemática. 


\section{ABSTRACT}

The called Riemann Zeta function was presented by Euler like the function $\xi(z)=\sum_{n=1}^{\infty} \frac{1}{n^{2}}$, that is a convergent series where $z$ is a complex number with positive real part. The calculation of series in the manner $\sum_{n=1}^{\infty} \frac{1}{n^{2 k}}$ is not found in general way in books or other investigation materials. Usually, you can find particular cases, i.e., basic examples. The present work goes guided in order to present a recurrent formula for the calculation of these series. For the understanding of this writing, the reader should have some notions, as they are it: function analytic, sequential expansion of Taylor, series of Laurent, among others.

Key words: Laurent's Serie, Bernoulli's numbers and mathematical induction.

\section{INTRODUCCIÓN}

La serie armónica generalizada, o serie $\mathrm{p}$, es alguna de las series $\sum_{n=1}^{\infty} \frac{1}{n^{p}}$, donde $p \in R^{+}$. La serie converge si $p>1$ y diverge en otro caso. En particular, si $p=1$, la serie es armónica. Si $p>1$, entonces la suma de la serie es $\xi(p)$, es decir, la función zeta de Riemann evaluada en $p$, [3], [5].

\section{MARCO TEÓRICO}

La expansión de Laurent [1], de $\left(e^{z}-1\right)^{-1}$ en el origen tiene la forma $\frac{1}{z}-\frac{1}{2}+\sum_{k=1}^{\infty}(-1)^{k-1} \frac{B_{k}}{(2 k) !} z^{2 k-1}$ donde $B k$ son los números de Bernoulli. Usaremos este hecho para sacar una fórmula de recurrencia a fin de encontrar algorítmicamente los números de Bernoulli.

Desde ahora, utilizaremos la división larga cada vez que sea necesario, i.e. si

$$
\begin{aligned}
\frac{a+b}{e^{z}-1}=\frac{a}{z}+ & \frac{b-a \sum_{k=2}^{\infty} \frac{z^{k-1}}{k !}}{e^{z}-1} \text {. Luego: } \\
& \left(e^{z}-1\right)^{-1}=\frac{1}{e^{z}-1}=\frac{1}{\sum_{k=2}^{\infty} \frac{z^{k}}{k !}}=\frac{1}{z}+\frac{-\sum_{k=2}^{\infty} \frac{z^{k-1}}{k !}}{e^{z}-1}=\frac{1}{z}+\frac{\frac{-z}{2}-\sum_{k=3}^{\infty} \frac{z^{k-1}}{k !}}{e^{z}-1} \\
= & \frac{1}{z}-\frac{1}{2}+\frac{-\sum_{k=2}^{\infty} z^{k}\left(\frac{1}{2 k !}-\frac{1}{(k+1) !}\right)}{e^{z}-1}=\frac{1}{z}-\frac{1}{2}+\frac{z^{2}\left(\frac{1}{2.2 !}-\frac{1}{3 !}\right)+\sum_{k=3}^{\infty} z^{k}\left(\frac{1}{2 k !}-\frac{1}{(k+1) !}\right)}{e^{z}-1} \\
= & \frac{1}{z}-\frac{1}{2}+\frac{z}{12}+\frac{\sum_{k=3}^{\infty} z^{k}\left(\frac{1}{2 k !}-\frac{1}{(k+1) !}\right)-\sum_{k=2}^{\infty} \frac{z^{k+1}}{k !} \frac{B_{1}}{2}}{e^{z}-1} \text { donde } \frac{z}{12}=\frac{B_{1}}{2} z \\
= & \frac{1}{z}-\frac{1}{2}+\frac{z}{12}-\frac{z^{3}}{30.4 !}+\frac{\sum_{k=5}^{\infty} z^{k}\left(\frac{1}{2 k !}-\frac{1}{(k+1) !}-\frac{B_{1}}{2(k-1) !}+\frac{B_{2}}{4 !(k-3) !}\right)}{e^{z}-1}
\end{aligned}
$$


[Nótese que para $k=5$ el valor es cero]

Con una simple inspección de la ecuación (1) obtenemos que

$$
B_{1}=\frac{1}{6}, B_{2}=\frac{1}{30} \text { y } B_{3}=\frac{1}{42}
$$

Observando detenidamente (1), podemos intuir la siguiente relación de recurrencia para calcular un número de Bernoulli a partir de los números de Bernoulli anteriores. Esta relación es:

$$
(-1)^{k+1} \frac{B_{k}}{(2 k) !}=\frac{1}{2 \cdot(2 k) !}-\frac{1}{(2 k-1) !}+\sum_{j=1}^{k-1} \frac{(-1)^{j} B_{j}}{(2 j) !(2 k-2 j+1) !}
$$

A continuación, probaremos esta igualdad usando inducción sobre k. Por hipótesis de inducción, se supone que:

$$
\left(e^{z}-1\right)^{-1}=\frac{1}{z}-\frac{1}{2}+\sum_{l=1}^{k} \frac{(-1)^{l-1)} B_{l} z^{2 l-1}}{(2 l) !}+\frac{\sum_{m=2 k+1}^{\infty}\left[\frac{1}{2 m !}-\frac{1}{(m+1) !}+\sum_{j=1}^{k} \frac{(-1)^{j} B_{j}}{(2 j) !(m-2 j+1) !}\right] z^{m}}{e^{z}-1}
$$

$$
=\frac{1}{z}-\frac{1}{2}+\sum_{l=1}^{k} \frac{(-1)^{l-1} B_{l} z^{2 l-1}}{(2 l) !}+\frac{\left[\frac{1}{2(2 k+2) !}-\frac{1}{(2 k+3) !}+\sum_{j=1}^{k} \frac{(-1)^{j} B_{j}}{(2 j) !(2 k-2 j+3) !}\right] z^{2 k+2}}{e^{z}-1}
$$

$$
+\frac{\sum_{m=2 k+3}^{\infty}\left[\frac{1}{2 m !}-\frac{1}{(m+1) !}+\sum_{j=1}^{k} \frac{(-1)^{j} B_{j}}{(2 j) !(2 k-2 j+1) !}\right] z^{m}}{e^{z}-1}
$$

Ahora, haciendo división larga se obtiene:

$$
\begin{aligned}
& \left(e^{z}-1\right)^{-1}=\frac{1}{z}-\frac{1}{2}+\sum_{l=1}^{k} \frac{(-1)^{l-1)} B_{l} z^{2 l-1}}{(2 l) !} \\
& +\frac{\sum_{m=2 k+1}^{\infty}\left[\frac{1}{2 m !}-\frac{1}{(m+1) !}+\sum_{j=1}^{k} \frac{(-1)^{j} B_{j}}{(2 j) !(m-2 j+1) !}+\frac{(-1)^{k} B_{k+1}}{(2 k+2) !(m=2 k-1) !}\right] z^{m}}{e^{z}-1}
\end{aligned}
$$




$$
=\frac{1}{z}-\frac{1}{2}+\sum_{l=1}^{k+1} \frac{(-1)^{l-1)} B_{l} z^{2 l-1}}{(2 l) !}+\frac{\sum_{m=2 k+1}^{\infty}\left[\frac{1}{2 m !}-\frac{1}{(m+1) !}+\sum_{j=1}^{k+1} \frac{(-1)^{j} B_{j}}{(2 j) !(m-2 j+1) !}\right] z^{m}}{e^{z}-1}
$$

Así, hemos obtenido la relación de recurrencia para los números de Bernoulli:

$$
B_{k}=(-1)^{k-1}\left[\frac{1}{2}-\frac{1}{2 k+1}+\sum_{l=1}^{k-1}(-1)^{l}\left(\begin{array}{l}
2 k \\
2 j
\end{array}\right) \frac{B_{l}}{2 k-2 j+1}\right]
$$

Ahora, expresaremos el desarrollo de Laurent de cot z en términos de los números de Bernoulli (para comparar estos coeficientes con los obtenidos en la expresión por sumas parciales).

Recordemos que:

$$
\begin{aligned}
& e^{z}=\cos z+i \operatorname{sen} z, \\
& e^{-i z}=\cos z-i \operatorname{sen} z, \\
& \frac{e^{i z}+e^{-i z}}{2}=\cos z \quad \text { y } \quad \frac{e^{i z}-e^{-i z}}{2 i}=\operatorname{sen} z ; \quad \text { de esta forma, } \\
& \cot z=\frac{\cos z}{\operatorname{sen} z}=\frac{\frac{e^{i z}+e^{-i z}}{2}}{\frac{e^{i z}-e^{-i z}}{2 i}}=i\left(\frac{e^{i z}+e^{-i z}}{e^{i z}-e^{-i z}}\right)=i\left(\frac{e^{2 i z}+1}{e^{2 i z}-1}\right)=1+\frac{2}{e^{2 i z}-1}=i\left[1+2\left(e^{i z}-1\right)^{-1}\right]
\end{aligned}
$$

Pero como se puede expresar:

$$
\begin{aligned}
& \left(e^{i z}-1\right)^{-1}=\frac{1}{z}-\frac{1}{2}+\sum_{k=1}^{\infty} \frac{(-1)^{k-1} B_{k}}{(2 k) !} z^{2 k-1} \\
& \cot z=\frac{1}{z}-\sum_{k=1}^{\infty} \frac{4^{k} B_{k} z^{2 k-1}}{(2 k) !}
\end{aligned}
$$

Se verifica que $\pi \cot (\pi . z)=\frac{1}{z}+\sum_{n \neq 0}\left(\frac{1}{z-n}+\frac{1}{n}\right)$ [1]. Estos resultados se utilizarán para comparar los coeficientes en el desarrollo de Laurent de $\cot (\pi . z)$ y de su expresión por sumas parciales y encontraremos, la expresión de $\sum_{n=1}^{\infty} \frac{1}{n^{2 k}}$ 
Se comienza trabajando sobre la ecuación (4). Haciendo un pequeño cambio se observa que:

$$
\cot (\pi \cdot z)=\frac{1}{\pi \cdot z}-\sum_{k=1}^{\infty} \frac{4^{k} B_{k}(\pi \cdot z)^{2 k-1}}{(2 k) !}
$$

Igualando estas dos últimas ecuaciones obtenemos:

$$
\sum_{n \neq 0}\left(\frac{1}{z-n}+\frac{1}{n}\right)=-\pi \sum_{k=1}^{\infty} \frac{4^{k} B_{k}}{(2 k) !}(\pi \cdot z)^{2 k-1}
$$

Ya que la convergencia de éstas dos series es uniforme [2] (por comparación con $\sum \frac{1}{n^{2}}$, se deriva término a término para obtener:

$$
\sum_{n \neq 0} \frac{-1}{(z-n)^{2}}=-\pi\left[\left[\frac{4 B_{1} \pi}{2 !}+\sum_{k=2}^{\infty} \frac{4^{k} B_{k}}{(2 k) !}(2 k-1) \pi(\pi . z)^{2 k-2}\right]\right]
$$

Derivando término a término (Teorema de Weierstrass, ver Ahlfors [1]), dos veces más, se obtiene:

$$
\sum_{n \neq 0} \frac{5 !}{(z-n)^{6}}=\pi^{6}\left[5 ! \frac{4^{3} B_{3}}{6 !}+\sum_{k=4}^{\infty} \frac{4^{k} B_{k}}{(2 k) !}(2 k-1)(2 k-2)(2 k-3)(2 k-4)(2 k-5)(\pi . z)^{2 k-6}\right]
$$

Ahora en la ecuación (6) la evaluamos en z=0, y así:

$$
\begin{aligned}
& \sum_{n \neq 0} \frac{-1}{(0-n)^{2}}=-\pi\left[\frac{4 B_{1}}{2 !}\right], \text { donde } B_{1}=\frac{1}{6} \\
& \sum_{n \neq 0} \frac{-1}{n^{2}}=-\pi^{2} 2\left[\frac{1}{6}\right], \text { luego } \\
& 2 \sum_{n \neq 0} \frac{1}{n^{2}}=\left[\frac{2 \pi^{2}}{6}\right], \text { es decir, } \sum_{n \neq 0} \frac{1}{n^{2}}=\left[\frac{\pi^{2}}{6}\right]
\end{aligned}
$$

Haciendo lo mismo en la ecuación (7) y (8), es decir, evaluar estas en z=0, se obtiene:

$$
\begin{aligned}
& \sum_{n=1}^{\infty} \frac{1}{n^{4}}=\frac{\pi^{4}}{90}, \mathrm{y} \\
& \sum_{n=1}^{\infty} \frac{1}{n^{6}}=\frac{\pi^{6}}{945}
\end{aligned}
$$


Desde la ecuación (8) se espera cumplir la siguiente igualdad:

$$
\sum_{n \neq 0} \frac{-(2 k-1) !}{(z-n)^{2 k}}=-\pi^{2 k}\left[\frac{4^{k} B_{k}(2 k-1) !}{(2 k) !}+\sum_{k=2}^{\infty} \frac{4^{j} B_{j}(2 j-1) \ldots(2 j-(2 k-1))}{(2 j) !}(\pi . z)^{2 j-2 k}\right]
$$

En efecto, derivando dos veces se tiene:

$$
\begin{gathered}
\sum_{n \neq 0} \frac{-(2 k) !}{(z-n)^{2 k+1}}=-\pi^{2 k+1}\left[\begin{array}{l}
\frac{4^{k+1} B_{k+1}(2 k-1) !}{(2(k+1)) !}(2 k+1)(2 k)(2 k-1) \ldots(3)(2)(\pi z)+ \\
\sum_{j=k+2}^{\infty} \frac{4^{j} B_{j}(2 j-1) \ldots(2 j-(2 k-1)(2 j-2 k))}{(2 j) !}(\pi . z)^{2 j-2 k-1} \pi
\end{array}\right] \mathrm{y} \\
\sum_{n \neq 0} \frac{-(2 k) !}{(z-n)^{2 k+1}}=-\pi^{2 k+1}\left[\begin{array}{l}
\frac{4^{k+1} B_{k+1}(2 k-1) !}{(2(k+1)) !}(2 k+1)(2 k)(2 k-1) \ldots(3)(2)(\pi z)+ \\
\sum_{j=k+2}^{\infty} \frac{4^{j} B_{j}(2 j-1) \ldots(2 j-(2 k-1)(2 j-2 k))}{(2 j) !}(\pi . z)^{2 j-2 k-1} \pi
\end{array}\right]
\end{gathered}
$$

Cuyo resultado es el mismo de la ecuación (9) cambiando k por $k+1$. Si z = 0 en la ecuación (9) se muestra que:

$$
\sum_{n=1}^{\infty} \frac{1}{n^{2 k}}=\frac{\pi^{2 k} 4^{k} B_{k}}{2(2 k) !}
$$

Donde Bk son los números de Bernoulli.

\section{VERIFICACIÓN EN MAPLE}

Lo primero que se hará, será hallar los primeros 10 números de Bernoulli; utilizando el programa Maple11.01:

$$
\begin{gathered}
\left.>\operatorname{seq}\left(\text { abs(bernoulli( }\left({ }^{*} \mathbf{n}\right)\right), \mathbf{n}=1 . .10\right) ; \\
\frac{1}{6}, \frac{1}{30}, \frac{1}{42}, \frac{1}{30}, \frac{5}{66}, \frac{691}{2730}, \frac{7}{6}, \frac{3617}{510}, \frac{43867}{798}, \frac{174611}{330}
\end{gathered}
$$


Se define la función B(j), de la siguiente manera,

\section{$>$ B:=j->abs(bernoulli(2*j)); \\ $B:=j \rightarrow$ abs|bernoulli $(2 j) \mid$}

Esta fórmula, permite obtener los números de Bernoulli mostrados anteriormente, utilizando una función incorporada ya en el software Maple11.01. A continuación, se muestra que se logran idénticos resultados, a través de la forma general para el cálculo de $\sum_{n=1}^{\infty} \frac{1}{n^{2 k}}$ así:

$$
\begin{aligned}
& B_{k}=(-1)^{k-1}\left[\frac{1}{2}-\frac{1}{2 k+1}+\sum_{l=1}^{k-1}(-1)^{l}\left(\begin{array}{l}
2 k \\
2 j
\end{array}\right) \frac{B_{l}}{2 k-2 j+1}\right] \\
& B_{k}:=k->(-1)^{k-1}\left[\frac{1}{2}-\frac{1}{2 k+1}+\sum_{l=1}^{k-1}(-1)^{l}\left(\begin{array}{l}
2 k \\
2 j
\end{array}\right) \frac{B_{l}}{2 k-2 j+1}\right] \\
& B:=j \rightarrow \text { abs|bernoulli(2j)| } \\
& \text { > B(1); } \\
& 1 \\
& 6 \\
& >\mathrm{B}(2) \text {; } \\
& \frac{1}{30} \\
& \text { > B(10); } \\
& \underline{174611} \\
& 330
\end{aligned}
$$

Si observamos que los números arrojados por la función $B(k)$, son los mismos que los números de Bernoulli. Con el resultado obtenido $\mathrm{B}(\mathrm{k})$; se genera el resultado $\mathrm{S}(\mathrm{k})$ el cual dará el cálculo de una serie de la forma $\sum_{n=1}^{\infty} \frac{1}{n^{2 k}}$

$$
\frac{174611}{330}
$$

$>\mathrm{S}:=\mathrm{j}->\left(\left((p i)^{\wedge}\left(2^{*} \mathrm{j}\right)^{*}(4)^{\wedge}(\mathrm{j})^{*} \mathrm{~B}(\mathrm{j})\right) /\left(2^{*}\left(2^{*} \mathrm{j}\right) !\right)\right.$

$$
S:=j \rightarrow \frac{1}{2} \frac{\pi^{2 j} 4^{j} B(j)}{(2 j) !}
$$


Para comparar es necesario obtener algunos resultados:

$$
\begin{gathered}
>\mathbf{S}(1) ; \frac{1}{6} \pi^{2}(14) \\
>\mathbf{S}(2) ; \frac{1}{90} \pi^{4}(15) \\
>\mathrm{S}(3) ; \frac{1}{945} \pi^{6}(16) \\
>\mathrm{S}(\mathbf{1 0}) ; \frac{174611}{1531329465290625} \pi^{20}
\end{gathered}
$$

Se define la serie $s(k)$ y se obtienen algunos resultados:

$$
\begin{aligned}
>\mathrm{s}:=\mathrm{k}->\operatorname{sum}( & \left.\mathbf{1} /(\mathbf{n})^{\wedge}\left(2^{*} \mathbf{k}\right), \mathbf{n}=\mathbf{1} . . \text { infinity }\right) \\
s & :=k \rightarrow \sum_{n=1}^{\infty} \frac{1}{n^{2 k}} \\
& >\mathrm{s}(\mathbf{1}) ; \frac{1}{6} \pi^{2} \\
& >\mathrm{s}(\mathbf{2}) ; \frac{1}{90} \pi^{4} \\
& >\mathbf{s}(\mathbf{3}) ; \frac{1}{945} \pi^{6} \\
>\mathbf{s}(\mathbf{1 0}) ; & \frac{174611}{1531329465290625} \pi^{20}
\end{aligned}
$$

Como se observa las dos funciones $S(k)$ y $s(k)$ generan los mismos resultados para los valores asignados a $\mathrm{k}$; Con lo cual queda comprobado computacionalmente los resultados obtenidos en el artículo.

\section{CONCLUSIÓN}

Se encontró una forma general para el cálculo de las series de la forma $\sum_{n=1}^{\infty} \frac{1}{n^{2 k}}$ a través de los números de Bernoulli. Estos a su vez, fueron obtenidos de una forma recurrente. Ambos resultados se escribieron después de ser demostrados por inducción 
matemática. La ecuación (10) se obtuvo matemáticamente, mientras que la ecuación (13) es obtenida por un medio computacional, verificando que los resultados son verdaderos.

\section{AGRADECIMIENTOS}

Agradecemos de una forma muy sincera, a la profesora Sandra Bello de la Universidad Militar Nueva Granada, por sus comentarios hechos a este escrito.

\section{REFERENCIAS}

[1] AHLFORS L. V. Complex Analysis. McGraw-Hill. New York 1966.

[2] APOSTOL TOM M. Análisis Matemático. Ed. Reverté S.A. Barcelona 2001.

[3] CHINEA Carlos. Divulgación de la matemática en la red. http://personales.ya.com/ casanchi/mat/zeta01.pdf.

[4] EDWARDS H M. Riemann's Zeta Function. Academic Press, 1974. New York.

[5] GOURDONX, SEBAHP. http://numbers.computation.free.fr/Constants/Miscellaneous/ zeta.html.

[6] SÁNCHEZ H. J. DARIO www.branchingnature.org/Variable Compleja Sánchez H.pdf. Última actualización: abril 2005.

[7] USER MANUAL. Maple 11. Maplesoft 2007. Canada. 
